as thiotepa is to miss one of the points of our communication ( 5 July, p 64), which is that bleomycin is an effective, safer agent to use as it does not cause myelosuppression and can therefore be given to those patients who have previously received aggressive cytotoxic chemotherapy. Less than $50 \%$ of the dose is absorbed and systemic effects are minimal. In addition, the inadvertent subcutaneous injection of bleomycin does not cause tissue necrosis and ulceration. It is for these reasons that we consider intracavitary bleomycin a safer agent for both inexperienced and experienced oncologists to administer. It is up to the individual to decide what price he pays for that safety.

Department of Radiotherapy and Oncology,

Norfolk and Norwich Hospital,

Norwich NR1 3SR

\section{Arterial puncture}

SIR,-I would like to comment on the article by Professor D C Flenley in "Procedures in Practice" dealing with arterial puncture (12 July, p 128). I would agree with him that puncture of the brachial artery is preferable to the radial since the procedure is not only rather less painful at the brachial artery but is less likely to give rise to arterial thrombosis. However, his diagrams show very clearly in two places that he prefers puncture in the antecubital fossa or, it would appear, just below.

My main objection to this site is that the artery, although easily palpable, is often restricted by the bicipital aponeurosis and any haematoma forming there may cause pressure on the median nerve. We had three such cases some four or five years $\mathrm{ago}^{1}$ and since then have carried out arterial puncture and cannulation about $2 \frac{1}{2} \mathrm{~cm}$ above the elbow crease or higher. In this site the artery is not tightly enclosed and we have had no further cases of median nerve damage A subsidiary point which does not come out of the article is that the brachial artery bifurcates at the region of the elbow.

Peter Sleight

\section{Cardiac Department, Oxford OX3 9DU}

${ }^{1}$ Littler WA. Postgrad Med $₹$ 1976;52, suppl 7:110-1.

SIR, - I read with interest the article by Professor D C Flenley on arterial puncture in the "Procedures in Practice" series (12 July, p 128). He describes the traditional technique of obtaining arterial blood, which I was taught as a medical student. This method, however, has numerous drawbacks.

Why, for example, is it necessary to use a green $(21 \mathrm{G})$ needle ? Such a large instrument is not only more painful but also causes more trauma to the artery than smaller needles. Perfectly adequate samples (even greater than the $10 \mathrm{ml}$ suggested) may be obtained with orange $(25 \mathrm{G})$ needles. A needle attached to a syringe may be difficult to manoeuvre while palpating the artery whereas that in the form of a "butterfly" may be gently inserted into the skin and manoeuvred over the vessel whence the transmitted pulsation of the vessel is easily seen moving the wings of the butterfly. The artery is then transfixed between index and middle finger while the other hand guides the needle into the vessel. The whole system- that is, butterfly and plastic syringe-should have its dead space filled with heparin and on puncture of the vessel arterial blood is seen pulsating in the clear plastic tubing of the butterfly connector, thus obviating the need for a glass syringe. Gentle suction on the syringe then fills it with blood.

The use of $25 \mathrm{G}$ needles enables the much more convenient radial artery to be punctured at the wrist. At this site palpation and trans fixion of the artery is easier over the underlyin bone. Indeed I have used this site on several occasions to obtain blood for reasons other than blood gas analysis in those such as the grossly obese in whom venepuncture is difficult if not impossible. The resultin haematoma is minimal provided constan pressure for five minutes is applied to the puncture site.

Professor Flenley claims that arterial puncture is a painful procedure requiring injection of local anaesthetic and that the - unbeliever should attempt it on himself. In the course of experiments my own radial artery has been punctured on several occasions. Each procedure was less painful than venepuncture and left a dull ache lasting a day over the site of the puncture.

Accident and Emergency

Department,

Guy's Hospital,

${ }_{*}^{*}$ We sent this letter to Professor Flenley, who replies as follows.-ED, $B M$ f.

SIR,-I recommend arterial puncture using $21 \mathrm{G}$ (green) needle in the brachial artery on the basis of extensive experience over the last 20 years. I have occasionally used a $25 \mathrm{G}$ (orange) needle attached to a syringe, but have not found it easy to detect the pulsation of blood into the syringe (which is essential to identify that an artery, and not a vein, has been punctured) when using this smalle needle. I have no experience of a $21 \mathrm{G}$ needle on a "butterfly," but suspect that the smaller diameter of needle will still have this disadvantage.

The reasons for preferring the brachial artery are as expressed in my article-namely, the patient is familiar with blood sampling at this site; the artery is of a reasonable size and is easily palpable; and, most importantly, the larger volume of the cubital fossa can accumulate any haematoma without excessive pain. I can only repeat that my own experience of several radial punctures on myself has been more painful than my many brachial punctures. I note that even Dr Morice had a dull ache lasting for a day after radial puncture, which in my experience is very unusual following venepuncture. I fail to see any objection to the use of local anaesthetic if this as $\mathrm{Dr}$ Morice appears to agree, can diminish pain.

D C Flenley

Department of Respiratory

Medicine,

City Hospital,

\section{Proctoscopy and sigmoidoscopy}

SIR,-I was interested in the excellent article in your "Procedures and Practice" series by Mr D J Ellis and Mr P G Bevan (9 August, p 435).

I have found that one can do a most satis- factory sigmoidoscopy under intravenous diazepam in the ward without needing to take the patient to theatre or give any more potent anaesthetic, and would commend the procedure for various reasons. It is carried out in the left lateral position with the patient lying across the bed with the buttocks right to the edge.

Preparation is everything and patients should receive an enema on the preceding night followed by nothing by mouth after midnight (it is essential that well-intending night staff be prevented from giving a cup of tea to the sleepy patient). This means that a Beogex (sodium bicarbonate and anhydrous sodium acid phosphate) suppository at $7 \mathrm{am}$, the patient then emptying the bowel at 8 am and $9 \mathrm{am}$, can be followed by perfect visualisation at $10 \mathrm{am}$. The advantage is that the rectum is dry and normal and any inflammation is pathological and not induced by the cleansing agent. In my experience it is not satisfactory to do a sigmoidoscopy without bowel preparation, for although the rectum may be clear there are practically always faeces obstructing the view in the sigmoid.

With this method it is quite possible to complete the whole operation, including a biopsy if necessary, in about five minutes; and the diazepam will produce amnesia in the patient afterwards. One might view it as a medical rather than a surgical sigmoidoscopy.

R V STONE

Park Hospital,

Manchester M31 3SL

SIR,-We would like to take issue with $\mathrm{Mr} \mathrm{D} \mathrm{J}$ Ellis and Mr P G Bevan (9 August, p 435) where they advise a general anaesthetic for full visualisation of the lower sigmoid colon and rectum during sigmoidoscopy.

The rectosigmoid junction is negotiable in most patients, without anaesthetic, if an "adolescent" $(250 \times 16 \mathrm{~mm})$ sigmoidoscope is used. Usually only the anxious patients and those with rigidity of the sigmoid colon from diverticular disease pose any difficulty. The knee-elbow position facilitates negotiation of the rectosigmoid junction in these groups.

General anaesthetic does not facilitate the negotiation of the rectosigmoid junction and it carries a significantly greater risk of perforation of the colon from overinflation and injudicious advancement of the sigmoidoscope. It is also expensive in time and bed occupancy.

In those patients who do require a sigmoidoscopy under general anaesthetic we would still advise the use of the smaller sigmoidoscope, the larger one $(300 \times 19 \mathrm{~mm})$ being reserved for difficult biopsy and operative procedures.

ROBERT TALBOT RICHARD POCOCK

St James's Hospital,

Is it really a cerebrovascular accident?

SIR,-I was interested in Dr R L Bishton's letter (5 July, p 63). Professor Keith Simpson has this to say1: "Avoid ambiguities like 'birth injuries,' 'cerebrovascular accident,' or 'termination of pregnancy,' qualification of which must be given to make it clear that they were natural and not accidental or criminal occurrences." Later in the same paragraph he continues, "Indeed, vagueness of any kind is 
sure to result in the indignity of having the

SIR,-Having read Alison Wisbeach and supplied by the DHSS, although some local certificate refused by the registrar." The late Mr Gavin Thurston (who preferred in his office of coroner not to be referred to by his medical title) wrote, "Cerebral haemorrhage suggests head injury, possibly homicidal, to the registrar. Further inquiry usually reveals the more usual high blood pressure." In my own experience as a coroner many so-called cerebrovascular accidents or cerebral haemorrhages have turned out after investigation to be subdural haemorrhages due to injury.

Perhaps even worse is the latest horror"chronic obstructive airways disease": in my experience this is used for anything from asthma to carcinoma of the bronchus and was recently given as a cause of death for a coalminer with a $50 \%$ industrial disability pension for pneumoconiosis. He was survived by his widow, who would obviously not benefit from an inaccurate description of the reason for his demise.

F G HaILS

Staffordshire (North) Coroner's
District,

Stoke-on-Trent ST4 6AS

${ }^{1}$ Simpson K. Forensic medicine, 6th ed. London: Edward Arnold, 1969:208.

2 Thurston G. Coroner's practice. London: Butterworth, 1958:30.

\section{Children in wheelchairs} Professor K S Holt's article "Children in wheelchairs" (19 July, p 199), I wondered if the Ortho-Kinetic chair had been considered. We find it answers many problems, especially transfer to the car. The chair becomes the car seat, with no adaptations to the car necessary and no lifting of the patients involved.

It is aesthetically pleasing and has many adaptations. Its main drawbacks are that it is only attendant operated and it is not yet authorities are interested.

Pat Freeman Superintendent Physiotherapist, Lewisham Health District

Beckenham, Kent

under stress and in cases of painful metastases in bone. When designed the wheat-grain beds and cushions were intended for short-term use, but they proved very popular also in longterm use, despite their obvious disadvantages. As soon as I was able to obtain the expanded polystyrene beads to take the place of the wheat grains their lightness, warmth, and simplicity of laundering were appreciated: while the clinical effectiveness in providing a dependable and entirely comfortable medium of support eliminated the instability inherent in all forms of spring support and uncontrolled fluid media such as water and air.

Much small-team work was involved in the design and production of a generally useful and acceptable series of "granular support cushions" -neck cushions, bed cushions (from shoulders to knees), and seat cushions (for most seating problems, including driving all sorts of vehicles, and especially appreciated by the "chair-fast" for comfort and stability, preventing and healing pressure sores in difficult cases). Finally, granular support heel and calf cushions were designed at the specific request of one of the three Exeter orthopaedic surgeons, all of whom now constantly use them for fracture patients on traction in slings in practically all cases since they were introduced some three years ago.

These and the other types mentioned are now in regular use in three Exeter hospitals, by many of my own patients and those of GP colleagues, and by the welfare services of area health authorities; and they have been supplied to Cheshire homes and to the Marie Curie Memorial and other establishments.

Having developed them from personal experience of their stabilising properties for back and neck troubles, I have slept continuously on these cushions during the last 10 years and take them everywhere-they should be more widely known and used.

JOHN B TRACEY

Pinhoe, Exeter

1 Tracey JB. Practitioner 1970;204:845-6.

Tracey JB. Handbook of impact therapy. 3rd ed, 1979 supplement. Personal publication, 1979:102-10.

\section{Treatment of axillary hyperhidrosis}

\section{Seating the disabled} (5 April, p 963) "Seating the disabled," in my experience this is not necessarily a complicated problem, provided that basic principles of support are fulfilled-namely, maximum area of base support for best stability and balance complete adaptation to the part supported; and a limited variability that can be produced at will to ensure comfort.

"Granular support," a term used to cover this type of supportive medium, was first used by me some 20 years ago to fulfil these criteria and so incidentally to eliminate "pressure points." In a suitably designed cushion, the friction between the granules must be sufficiently low to ensure full adaptation of the cushion to the bodily contours, in the first instance by voluntary movements of adjustment, but of a sufficiently high friction to retain the chosen position of comfort, which can be varied at will. I have described ${ }^{12}$ a welltried and simple application of these principles that gave great relief to sufferers both from seat troubles and from back pain, among many other applications in the support of joints by Mr K T Scholes and others (8 July 1978 , p 84) and by Mr C R W Rayner and others (10 May, p 1168), and the subsequent correspondence culminating in the rejoinder from $\mathrm{Mr}$ Colin Rayner to Dr W F G Tucker's letter (16 August, p 520). As my company markets a formulation of aluminium chloride hexahydrate (Anhydrol Forte) corresponding to that used by $\mathrm{Mr}$ Scholes and his colleagues I am sure your readers will be interested in the following factual observations-free, I trust, from any commercial bias.

In the first place, we absolutely confirm $\mathrm{Mr}$ Scholes's statement that it takes three weeks to prepare the $20 \%$ solution of aluminium chloride hexahydrate in alcohol. Any attempt to shorten this time results in a portion of the crystals failing to dissolve and the supernatant liquid being of a lower concentration. This has occasionally happened in hospital pharmacies, owing to the difficulty in compounding this formulation, and we have examined some samples purporting to be of full strength, but on analysis proving to have much lower levels-one as low as $12 \%$.
SIR,-With reference to your leading article
Much more important, however, is the fact that almost always patients will use far too much of the solution if applying it with a brush. We found in our preliminary clinical trials that severe local reactions occurred to the extent described by $\mathrm{Mr}$ Rayner and that these reactions almost completely disappeared when a roll-on applicator (which permits only a tiny instead of a brush. This is borne out by the fact that since issuing Anhydrol Forte in June no adverse reactions have been reported.

Incidentally, I cannot understand Dr Tucker's reference to "an aqueous solution of $20 \%$ aluminium chloride hexahydrate." It is well known that aluminium chloride hydrolyses in aqueous solution to form hydrochloric acid; that is why the injunction to make sure that the site is absolutely dry before the solution is applied must be observed if irritation is to be avoided.

Dermal Laboratories Limited

H YARROW

Hitchin, Herts SG4 7QR

\section{Aortic stenosis from calcification of congenital bicuspid valve}

SIR,-Dr N Brooks's report (9 August, p 424) on cases of rapid development of severe aortic stenosis points out the difficulties encountered by physicians in assessing the degree of stenosis clinically and sometimes even haemodynamically.

Although cases 2 and 3 showed an initial small transvalvar gradient, despite the use of the same equipment, this does not necessarily reflect insignificant stenosis haemodynamically. Heart rate and flow can be extremely variable and with a valve whose area is narrowed by around $50 \%$ may still show an insignificant pressure gradient. This results from the relationship between the flow and gradient for a particular valve area which shows a gradually diminishing slope until it becomes almost flat. Furthermore, with a very narrow valve area the curve becomes an asymptote exponential reaching a plateau at very low flows. The difference in heart rate between any two investigations carried out at different times is also very important because even though the cardiac output may be identical the systolic

SIR,-I have followed with great interest the ejection period will be different as the rate papers on treatment of axillary hyperhidrosis changes.

If on clinical grounds there are indication for catheterising patients with aortic stenosis and an "insignificant" gradient is discovered, concomitant cardiac output measurement across the valve or pressure gradient measurement following a period of exercise (or both) is indicated as a small change in flow demands a large increase in pressure gradient; this relationship is more marked the narrower the valve area. Such manoeuvres may bring out a more realistic index of obstruction than resting pressure gradients alone.

J V DE GIOvanNI

Birmingham General Hospital, Birmingham

\section{The motives of fakers}

SIR,-Sir Douglas Hubble (16 August, p 523) has seriously underestimated the number of fakers exposed in the past 10 years. To his list we may add the names of Summerlin, Derr, ${ }^{2}$ and Gullis ${ }^{3}$ in the biological field alone. calculated amount to be applied) was used 